\title{
Post-traumatic osteolysis of the clavicle: a case report
}

\author{
C. J. GRIFFITHS AND E. GLUCKSMAN
}

Department of Accident and Emergency Medicine, King's College Hospital, Denmark Hill, London, England

\section{SUMMARY}

A case of post-traumatic osteolysis of the clavicle occurring in a 25-year-old male is described. The condition should be suspected in anyone giving a history of persistent shoulder pain following trauma or intensive sporting activities. Characteristic resorption of the distal tip of the clavicle is found on X-ray. The condition usually responds within 2 years to conservative treatment. Previous reports of the condition are reviewed, and the possible pathogenesis and differential diagnosis discussed.

\section{INTRODUCTION}

Post-traumatic osteolysis of the clavicle is an uncommon condition characterized by persistent shoulder pain associated with decalcification of the distal tip of the clavicle following trauma to the acromio-clavicular joint (Madsen, 1963; Smart, 1972; Quinn \& Glass, 1983). Patients usually give a history of previous trauma to the shoulder, but the condition may follow active sports such as weight training (Cahill, 1982) or the use of pneumatic tools (Ehricht, 1959). The condition usually resolves within 2 years in the absence of further trauma. This article describes a case of post-traumatic osteolysis of the clavicle, and discusses the diagnosis and possible pathogenesis of the condition.

\section{CASE REPORT}

A 25-year-old medical student (C.G.) was involved in a bicycle accident and received a direct blow to his right shoulder. On examination, shoulder movements were found to be full but painful. X-ray revealed no injury to either bone or joint (Fig. 1a) and the

Correspondence: $\operatorname{Dr}$ E. Glucksman M.D., M.R.C.P., Consultant in Accident and Emergency, King's College Hospital, Denmark Hill, London, SEs $9 R$ S, England 

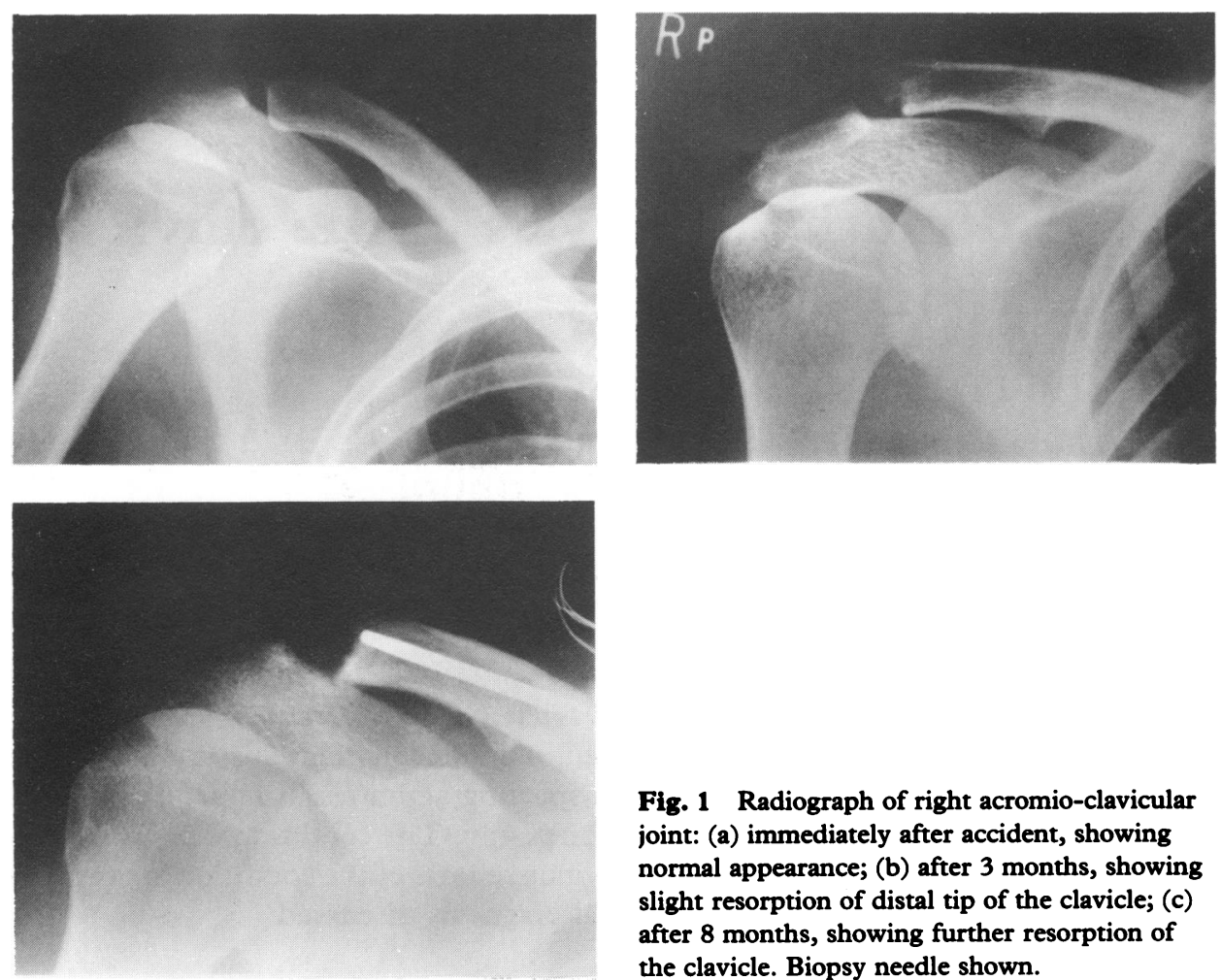

Fig. 1 Radiograph of right acromio-clavicular joint: (a) immediately after accident, showing normal appearance; (b) after 3 months, showing slight resorption of distal tip of the clavicle; (c) after 8 months, showing further resorption of the clavicle. Biopsy needle shown.

patient was discharged. Over the next 6 months, the shoulder remained painful, particularly during active movement and when lying on the affected side. Examination 3 months after the injury revealed point tenderness and deformity over the right acromioclavicular joint. X-ray showed subluxation of the acromio-clavicular joint and slight resorption of the distal end of the clavicle (Fig. 1b). X-ray examination 8 months after the injury showed further resorption, extending approximately $5 \mathrm{~mm}$ from the tip of the clavicle (Fig. 1c). A biopsy of the area 8 months after the accident showed patches of necrotic and reactive woven bone. Microbiological cultures of the biopsy were negative. Serum levels of calcium, phosphate and alkaline phosphatase were normal. Twelve months after the injury the shoulder had become virtually pain-free, although the radiological abnormalities remained.

\section{DISCUSSION}

According to Madsen (1963), post-traumatic osteolysis of the clavicle was first described by Werder (1950). Although about a hundred cases have been reported, the condition is infrequently recognized and its pathology remains unclear. The great majority of cases have occurred in males. The characteristic features of the condition are 
persistent shoulder pain, weakness of shoulder movement, with swelling and tenderness over the acromio-clavicular joint. These are accompanied by radiological evidence of osteolysis of the distal tip of the clavicle. Other features may include joint crepitus, ossification of the acromio-clavicular and coraco-clavicular ligaments, and occasional osteolytic involvement of the acromion (Cahill, 1982; Levine et al., 1976). Patients often give a history of a single episode of trauma to the shoulder but the condition has also been described in the absence of acute trauma, notably in male athletes (Smart, 1972; Cahill, 1982), and following the use of pneumatic tools (Ehricht, 1959). Although usually unilateral, bilateral osteolysis has been reported, notably amongst athletes (Smart, 1972; Cahill, 1982).

In cases when a history of acute trauma is present, $\mathrm{X}$-ray immediately after the incident typically reveals only soft tissue swelling with an apparently normal clavicle. The acromio-clavicular joint may be intact or subluxed. Clavicular osteolysis is detectable radiologically as soon as $2 \frac{1}{2}$ weeks after the incident (Levine et al., 1976) and may progress for several years (Madsen, 1963). The delayed appearance of the radiological changes means that the condition may often be missed. Post-traumatic osteolysis has never been reported to involve more than $2 \mathrm{~cm}$ of the clavicle. Radiographs show resorption of the distal end of the clavicle due to loss of subarticular cortical bone, occasionally with cystic changes (Cahill, 1982; Orava et al., 1984). Similar radiological features may be apparent in the acromion (Levine et al., 1976).

Further trauma often induces a recurrence of shoulder pain but it is not clear if this is accompanied by further osteolysis. The condition usually resolves within a year with conservative treatment consisting of immobilization and the avoidance of further trauma. Resection of the distal clavicle has produced reliable relief of symptoms in athletes who persisted in training (Cahill, 1982). Remission of symptoms is concurrent with X-ray evidence of partial or complete reossification of the clavicle, although widening of the acromio-clavicular joint commonly remains. Levine et al. (1976) emphasize that early recognition of the condition with immediate immobilization may prevent progression of clavicular erosion.

The pathogenesis of the condition is not clear. A number of authors favour ischaemia as a cause. Orava et al. (1984) point out the resemblance of the condition to avascular necrosis of bone, a view supported by the appearance of the biopsy in the present case. Madsen (1963) proposed an autonomic mechanism producing a secondary alteration in blood supply, based on his finding of anisoscoria in four out of eight patients. However, anisoscoria does not appear to be a consistent finding in the published series. Similar osteolytic lesions occur in the phalanges following mechanical, electrical and thermal injury, after exposure to polyvinyl chloride monomers, and in association with rheumatoid conditions such as psoriatic arthritis and rheumatoid vasculitis (Destouet \& Murphy, 1983). These authors have suggested that vascular occlusion, produced by either thrombus or immune complex formation underlines the osteolysis that occurs in the phalanges in these cases. Cahill (1982) detected microfractures in $50 \%$ of clavicles resected from athletes; the resulting thrombus formation could produce ischaemia in the distal clavicle, so promoting a necrotic osteolysis in a way similar to that proposed for the phalanges.

An alternative explanation is proposed by Levine et al. (1976) who suggest that the process of osteolysis is secondary to synovial overgrowth. A resected acromio-clavicular 
joint showed hyperplastic fibrocartilage and synovium with vascular proliferation; resection was followed by reconstitution of the acromion.

The appearance of the clavicle in post-traumatic osteolysis must be distinguished $\frac{\vec{D}}{\mathbb{R}}$ from that seen occasionally in connective tissue disorders, hyperparathyroidism, $\stackrel{?}{\rightarrow}$ infection, neoplastic disease and massive osteolysis. In most cases, a history of trauma or $\stackrel{\vec{\rho}}{\stackrel{\rho}{\rho}}$ intensive sports activity together with the characteristic radiological findings are sufficient to make the diagnosis, particularly if radiographs taken immediately after injury are available showing an intact clavicle. Differentiation from hyperparathyroidism is made by the absence of generalized osteoporotic changes, of pathological fractures, and the characteristic alterations in serum levels of calcium, phosphate and parathyroid hormone. The connective tissue diseases such as rheumatoid and psoriatic arthritis may be excluded by the lack of other rheumatoid signs and typical serological findings. Infection would be associated with local inflammation, fever and a leucocytosis, while neoplastic disease, if consistent with the radiological findings, may best be excluded by biopsy of the affected clavicle. Massive osteolysis shows a relentlessly progressive course and more extensive skeletal distribution than does post-traumatic osteolysis.

\section{REFERENCES}

Cahill B. R. (1982) Osteolysis of the distal part of the clavicles in male athletes. Fournal of Bone and foin Surgery 64-A, 1053-8.

Destouet J. M. \& Murphy W. A. (1983) Acquired acroosteolysis and acronecrosis. Arthritis and Rheumatis 26, $1150-4$.

Ehricht H. G. (1959) Die Osteolyse im lateralen Claviculaende nach Pressluftschaden. Archiv fur orthopädische und Unfall-Chirurgie 50, 576-82.

Levine A. H., Pais M. J. \& Swartz E. E. (1976) Post-traumatic osteolysis of the distal clavicle with emphasis on early radiologic changes. American fournal of Roentgenology 17, 781-4.

Madsen B. (1963) Osteolysis of the acromial end of the clavicle following trauma. British fournal of Radiology 36, 822-8.

Orava S., Virtanen K. \& Holopainen Y. V. O. (1984) Post-traumatic osteolysis of the distal end of the clavicle. Annales Chirurgiae et Gynaecologiae Fenniae 73, 83-6.

Quinn S. F. \& Glass T. A. (1983) Post-traumatic osteolysis of the clavicle. Southern Medical fournal 76, 3078.

Smart M. J. (1972) Traumatic osteolysis of the distal ends of the clavicles. Fournal of the Canadian Association of Radiologists 23, 264-6.

Werder H. (1950) Posttraumatische Osteolyse des Schlusselbeinendes. Scweizerische Medizinische Woehenschrift 80, 912-3. 